\title{
Ulcerative jejunitis in a child with celiac disease
}

\author{
Terry Sigman ${ }^{1 *}$, Van-Hung Nguyen ${ }^{2}$, Florin Costea ${ }^{1}$, Ana Sant'Anna ${ }^{1}$ and Ernest G Seidman ${ }^{1}$
}

\begin{abstract}
Background: Celiac disease can present in children and adults with a variety of manifestations including a rare complication known as ulcerative jejunitis. The latter has been associated with refractory celiac disease in adult onset patients. The objective of this case report is to describe the first pediatric case of ulcerative jejunitis in celiac disease, diagnosed by capsule endoscopy, which was not associated with refractory celiac disease.

Case presentation: The 9 year old girl presented with a history of abdominal pain and vomiting. Laboratory investigations revealed a slightly elevated IgA tissue transglutaminase antibody level in the setting of serum IgA deficiency. Initial upper endoscopy with biopsies was not conclusive for celiac disease. Further investigations included positive IgA anti-endomysium antibody, and positive HLA DQ2 typing. Video capsule endoscopy showed delayed appearance of villi until the proximal to mid jejunum and jejunal mucosal ulcerations. Push enteroscopy with biopsies subsequently confirmed the diagnosis of celiac disease and ulcerative jejunitis. Immunohistochemical studies of the intraepithelial lymphocytes and PCR amplification revealed surface expression of CD3 and CD8 and oligoclonal T cell populations. A repeat capsule study and upper endoscopy, 1 year and 4 years following a strict gluten free diet showed endoscopic and histological normalization of the small bowel.

Conclusion: Ulcerative jejunitis in association with celiac disease has never previously been described in children. Capsule endoscopy was essential to both the diagnosis of celiac disease and its associated ulcerative jejunitis. The repeat capsule endoscopy findings, one year following institution of a gluten free diet, also suggest that ulcerative jejunitis is not always associated with refractory celiac disease and does not necessarily dictate a poor outcome.
\end{abstract}

Keywords: Ulcerative jejunitis, Celiac disease, Pediatric, Capsule endoscopy

\section{Background}

Celiac disease $(\mathrm{CD})$, or gluten-sensitive enteropathy, is an autoimmune disorder in which genetically susceptible individuals develop inflammatory changes in the small intestinal mucosa following the ingestion of gluten, a protein found in grains including wheat, rye and barley [1]. Clinical presentations vary extensively, from patients who are asymptomatic to those having one or more gastrointestinal symptoms such as abdominal pain, vomiting, diarrhea and bloating. Extraintestinal manifestations may include malnutrition and growth delay in children, osteoporosis, infertility, neurological symptoms and arthritis. Ulcerative jejunitis is a rare complication of $C D$ typically found in adults in the 5th or 6th decade. It is usually associated with refractory celiac disease (RCD)

\footnotetext{
* Correspondence: terry.sigman@muhc.mcgill.ca

'Division of Pediatric Gastroenterology, Montreal Children's Hospital, Faculty of Medicine, McGill University, 2300 Tupper St, Montreal, Quebec H3H 1P3, Canada

Full list of author information is available at the end of the article
}

and the possible development of enteropathy-associated T-cell lymphoma (EATL) [2,3]. Serological tests accurately identify those for whom a small bowel biopsy is indicated. The most specific serological tests available include IgA anti-endomysium (EMA) and IgA anti-tissue transglutaminase (tTG). The characteristic changes seen on small bowel biopsy include various degrees of villous atrophy with increased intraepithelial lymphocytes (IEL) and crypt hyperplasia, as summarized in the modified MarshOberhuber Classification criteria [4]. The classic criteria for diagnosis of $C D$ in a child with symptoms suggestive of $\mathrm{CD}$ include the characteristic histological changes on small bowel biopsy and clinical resolution after starting a gluten free diet (GFD) [1]. Positive serological tests that revert to normal following a GFD are supportive of the diagnosis [1]. Wireless capsule endoscopy of the small bowel (CE) is occasionally used as an adjunctive diagnostic test and has been shown to be helpful for detecting celiac disease [5]. CE provides high-resolution magnified views of the small intestinal mucosa and mucosal abnormalities
C Biomed Central

(c) 2014 Sigman et al.; licensee BioMed Central Ltd. This is an Open Access article distributed under the terms of the Creative Commons Attribution License (http://creativecommons.org/licenses/by/2.0), which permits unrestricted use, distribution, and reproduction in any medium, provided the original work is properly credited. 
include scalloping of folds, fissures or grooves, a mosaic pattern and absence or reduced duodenal folds [6].

In this case report we present the case of a child with ulcerative jejunitis associated with celiac disease that was diagnosed with $\mathrm{CE}$ and whose clinical symptoms, histological and capsule findings eventually resolved on a GFD.

\section{Case presentation}

A 9-year-old girl was referred for assessment of a 1 year history of episodic abdominal pain and non-bilious vomiting. The pain was described as dull, localized to the periumbilical area and unassociated with food. A breath test was consistent with lactose malabsorption. However, lactose restriction only transiently improved symptoms. The review of systems and past medical history were unremarkable. She denied the use medications including nonsteroidal anti-inflammatory drugs (NSAIDS). There was no family history of celiac disease, pancreatitis, inflammatory bowel disease, or autoimmune disorders other than multiple sclerosis (paternal grandmother). Her physical exam was within normal limits with satisfactory weight and growth parameters.

Initial laboratory investigations revealed a normal hemoglobin and white blood cell count, erythrocyte sedimentation rate and serum albumin. Her tTG was discretely elevated $(10 \mathrm{U} / \mathrm{ml}$; normal $<5)$. However, her total serum IgA was low $(<0.06 \mathrm{~g} / \mathrm{L}$; normal $0.63-3.49)$, whereas her IgE was elevated (986 ug/L; normal <240).

An initial upper endoscopy revealed small apthous ulcerations in the duodenal bulb. The second portion of the duodenum appeared normal. One duodenal biopsy revealed mild villous blunting and acute duodenitis, without an increased IEL count. No giardia or other parasites were seen. Two other duodenal biopsies revealed normal villi, with moderate eosinophlic infiltration involving the glands (40-50 eosinophils per high power field (HPF)). The pathology report concluded that the findings were insufficient for a diagnosis of celiac disease and that the focal eosinophilic infiltrates were consistent with infectious, allergic or eosinophilic gastroenteropathies. A colonoscopy revealed mild inflammatory changes in the terminal ileum and ileocecal valve as well as hyperemia of the mucosa in the rectosigmoid area. Histological analysis showed focal active ileitis, and foci of mild active colitis with intraepithelial eosinophils. No granulomas, crypt abscesses, or chronic inflammatory changes were seen. She was given a tentative diagnosis of eosinophilic gastroenteritis and was treated with once daily oral montelukast sodium. No sustained benefit was achieved.

In view of persistent symptoms, further investigations were pursued, which revealed positive EMA and HLA DQ2 typing. CE of the small bowel showed patchy villous atrophy with delayed appearance of villi until the proximal to mid jejunum, as well as areas with slight mucosal scalloping. Moreover, fibrin covered actively bleeding mucosal ulcerations were observed in the jejunum (Figure 1). Her tTG remained slightly elevated $(7 \mathrm{U} / \mathrm{ml}$; normal $<5)$. Push enteroscopy performed 7 months following her initial upper endoscopy, confirmed ulcerative jejunitis. Biopsies revealed subtotal villous atrophy, crypt hyperplasia and an IEL count of 58 lymphocytes/100 enterocytes in the second stage of the duodenum (Marsh classification III B). Changes in the jejunum varied from preserved villous architecture with $82 \mathrm{IEL} / 100$ enterocytes (Marsh I at $65 \mathrm{~cm}$ ) to partial villous atrophy and crypt hyperplasia with an IEL count of 89/100 enterocytes (Marsh III B at $95 \mathrm{~cm}$ ), consistent with a diagnosis of celiac disease (Figure 2).

Institution of a strict GFD for 6 months led to clinical improvement, with considerably less pain and cessation of vomiting. Moreover, her serum tTG normalized $(<3 \mathrm{U} / \mathrm{ml})$. However, a repeat $\mathrm{CE}$ again revealed delayed appearance of villi until the mid jejunum and mucosal ulcerations in the proximal jejunum without active bleeding, consistent with persistent ulcerative jejunitis. Biopsies obtained by repeat push enteroscopy after 6 months on a GFD showed normal villous architecture in the duodenum and jejunum down to $150 \mathrm{~cm}$, with an IEL count of 12/100 enterocytes per HPF in the duodenum (Marsh 0) and 40-46/100 per HPF in the jejunum at $130 \mathrm{~cm}$ and $150 \mathrm{~cm}$ respectively (Marsh I). The biopsies were subsequently sent for lymphocytic identification and PCR amplification studies for T cell rearrangement on fresh tissue. PCR amplification studies were also done on paraffin embedded tissue from the samples at diagnosis. On the sample from initial diagnosis prior to GFD, a T-cell clonal population was observed although it stained positive for surface expression of both CD3 and CD8 (Figure 3). The specimen obtained after 6 months on a GFD showed oligoclonal T-cell populations and still stained positive for surface expression of both CD3 and CD8 (Figure 4). Since there was definite improvement histologically, a decision was made to continue the strict GFD and to re-evaluate by $\mathrm{CE}$ and histology after another 6 months.

A third CE after 12 months on a GFD was unremarkable, with early appearance of normal villi in the duodenum and no evidence of ulcerative jejunitis. A duodenal biopsy now showed well preserved villous morphology and a normal IEL count (25 lymphocytes/100 enterocytes; CD3/CD8 positive), consistent with celiac disease in remission. A recent capsule, done 4 years after diagnosis, was unremarkable and multiple duodenal biopsies were completely normal as well. Her tTG remained normal $(<3 \mathrm{U} / \mathrm{ml})$, her EMA normalized and her total serum IgA remained low (0.4gL; normal 0.82-4.57).

\section{Discussion}

Most cases of $\mathrm{CD}$ are easily diagnosed with routine serological tests and duodenal biopsies. Unfortunately however, the diagnosis of celiac disease is not always straight forward. 


\section{Scalloping of distal} duodenal folds

2. Elevated mucosal lesion with broad villi
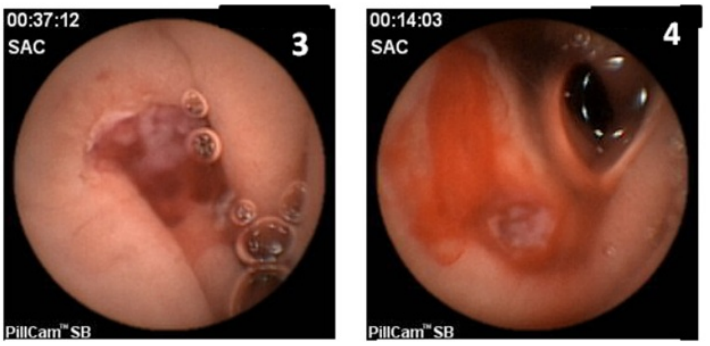

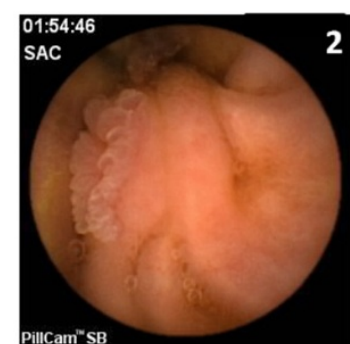

3. Fibrin covered and actively bleeding mucosal ulceration, jejunum.

4. Fibrin covered, actively bleeding mucosal ulceration in proximal jejunum; villous atrophy.

Figure 1 WIRELESS CAPSULE ENDOSCOPY Initial Exam (Normal Diet). Findings highly suggestive of celiac disease complicated by ulcerative jejunitis. Push enteroscopy suggested to obtain diagnosis and to rule out EATL.

Standard serological testing does not always identify the need for a small intestinal biopsy because CD occurs with a higher frequency in individuals with selective IgA deficiency, as in the present case [7]. A small percentage of patients with $\mathrm{CD}$ are seronegative [8]. Moreover, not all cases of $\mathrm{CD}$ have the classic histological criteria. Histological findings can vary due to patchy distribution or varying degrees of villous atrophy [4]. Furthermore, intraepithelial lymphocytosis, crypt hyperplasia and villous atrophy can be seen in other conditions affecting the small bowel, such as giardiasis, Crohn's disease, autoimmune and allergic enteropathies $[1,4,9]$. A diagnostic dilemma arose in this case in that the serology was borderline positive as the patient is IgA deficient. Additionally, the initial histopathological findings were not conclusive for $\mathrm{CD}$. We thus proceeded with $C E$ which revealed ulcerative jejunitis in addition to typical changes of $\mathrm{CD}$. This is consistent with the recent study [10] showing that $\mathrm{CE}$ is able to accurately diagnose villous atrophy in $\mathrm{CD}$ as well as complications such as ulcerative jejunitis.

Ulcerative jejunitis is rare, but can be seen in infections, or inflammatory conditions such as Crohn' disease or eosinophilic gastroenteritis, collagen vascular and other immune-mediated diseases such as vasculitis, systemic lupus erythematosis or Henoch-Schonlein purpura, as well as secondary to medications such as aspirin

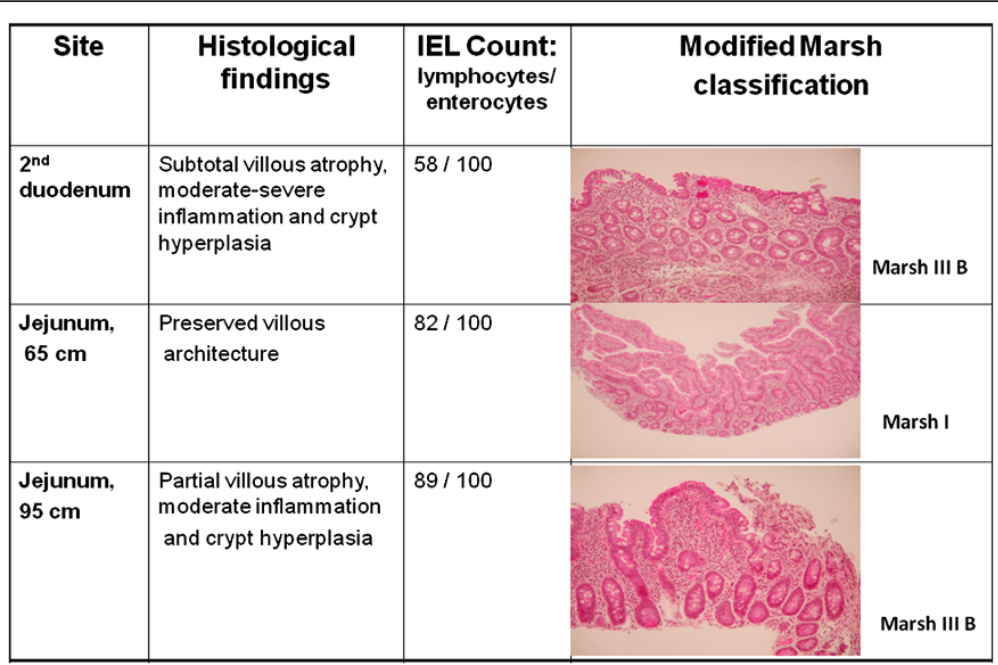

Figure 2 Histological findings on a normal diet. Histological confirmation of celiac disease by push enteroscopy. 


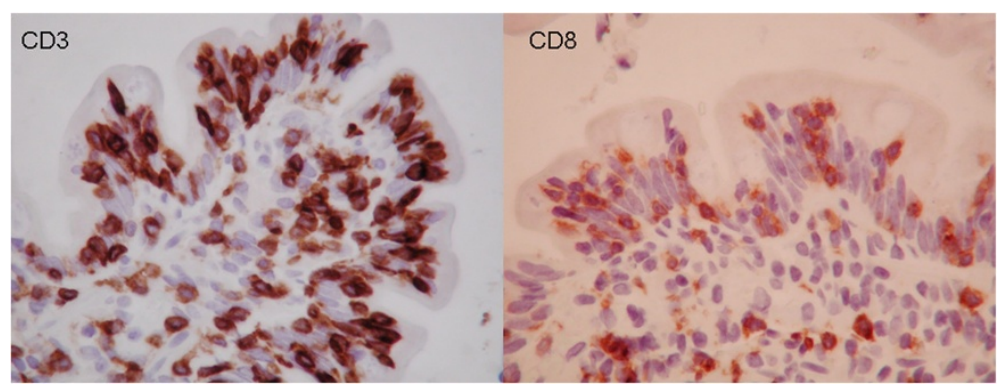

Figure 3 Immunohistochemical phenotyping of IELs (at $95 \mathrm{~cm}$ in jejunum) at time of diagnosis. Intraepithelial lymphocytosis (IEL), 89 lymphocytes/100 enterocytes with double positivity for CD3 (LEFT) and CD8 (RIGHT), and negative for CD4; consistent with Marsh III A classification.

or other NSAIDS, RCD, traumatic conditions such as intussusception, immunodeficiency disorders such as hypogammaglobulinemia or various neoplastic conditions [11]. In the pediatric literature, 4 cases of ulcerative jejunitis were diagnosed by $\mathrm{CE}$ in young children suffering from juvenile rheumatoid arthritits, three of which were attributed to NSAID use [12].

Among adult cases, ulcerative jejunitis is usually associated with RCD $[2,3]$. The definitions of RCD vary in the literature. RCD can be defined as either a primary nonresponse to a GFD or a secondary form, where patients have a relapse of their enteropathy despite a GFD [13]. Generally, RCD is diagnosed if there is no histological response to a GFD for more than 12 months or if severe symptoms persist necessitating intervention independent of duration of the GFD $[14,15]$. In fact, it was reported that it may take up to two years for histological recovery in a patient with uncomplicated CD on a GFD [14]. RCD is classified as types 1 and 2, depending on the surface expression of intraepithelial T-cells [16]. In type I RCD, the IEL express CD3, CD4 or CD8, CD103 and T-cell receptors (TCR) with polyclonal TCR gene rearrangement. These phenotypically normal IEL are indistinguishable from those seen in active, uncomplicated CD. In type II RCD, IEL lack surface CD3, CD4 or CD8, and TCR, but express intracytoplasmatic CD3 and surface CD103. The aberrant IEL in type II RCD are often characterized by monoclonal rearrangement of the TCR on the genomic level $[17,18]$. Such aberrant IEL have also been reported in ulcerative jejunitis and EATL, conditions that may co-exist or evolve from RCD [19]. These observations suggest that these disorders constitute a spectrum with type II RCD. A pediatric case of type I RCD was recently reported [20]. Although the clinical course is generally benign, type I RCD is associated with an increased risk of developing autoimmune disorders. Patients not responding to a strict GFD may benefit from immunosuppressive drugs [21,22], including thioguanine [23]. Type II RCD is associated with a risk of lymphoma and thus potentially a poor prognosis. Although no treatment has been found to be curative, chemotherapeutic agents are generally administered [24]. In severe cases, autologous stem cell transplantation has been employed for enteropathy associated T cell lymphoma [25].

The patient described herein is, to the best of our knowledge, unique in presenting with ulcerative jejunitis in association with $C D$ in the pediatric age group. Furthermore, the ulcerative jejunitis did not seem to be associated with RCD in this particular case. We did observe resolution of the ulcerative jejunitis as well as the enteropathy after 12 months on a GFD both histologically as well as shown by repeat $\mathrm{CE}$. Moreover, despite having ulcerative jejunitis, she never had abnormal T-cell phenotypes or evidence of EATL. In cases with ulcerative jejunitis, with or without RCD, a phenotypical analysis of IELs

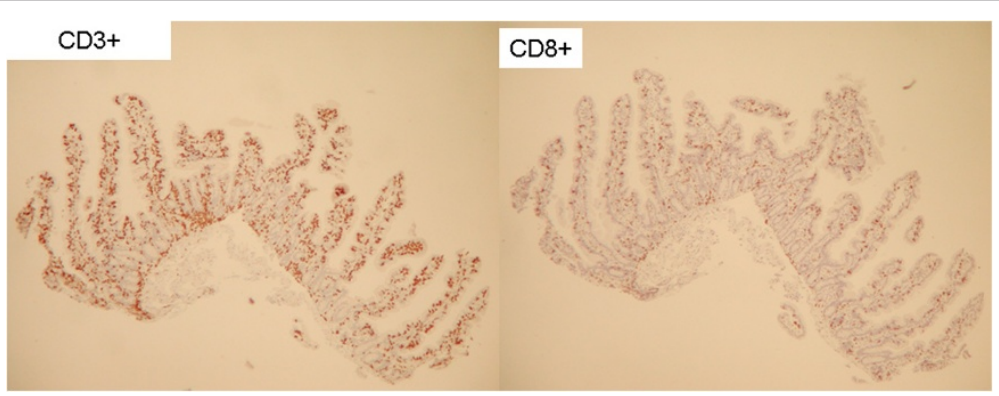

Figure 4 Histology and immunohistochemical phenotyping of IELs (at $150 \mathrm{~cm}$ in jejunum) 6 months after starting GFD. Intraepithelial lymphocytosis (IEL), 40 lymphocytes/100 enterocytes with double positivity for CD3 (LEFT) and CD8 (RIGHT), and negative for CD4; consistent with Marsh I classification. 
should be performed by immunohistochemistry, or if possible, using flow cytometry in order to distinguish between the two subtypes [26].

\section{Conclusion}

Ulcerative jejunitis in association with $\mathrm{CD}$ has never, based on our review of the literature, been described in the pediatric age group. CE was essential to establishing the initial diagnosis of $\mathrm{CD}$ in this patient and in identifying the associated ulcerative jejunitis, as well as its resolution. $\mathrm{CE}$ was also very helpful in guiding the need for tissue sampling and in following the outcome of ulcerative jejunitis complicating CD after institution of a GFD. The CE findings suggest that ulcerative jejunitis is not always associated with RCD, and as in this case, does not necessarily dictate a poor outcome. Further studies are needed to evaluate the incidence and outcome of ulcerative jejunitis and RCD in children with celiac disease.

\section{Consent}

Written informed consent was obtained from the patient for publication of this case report and any accompanying images. A copy of the written consent is available for review by the Editor of this journal. As the patient is a minor, parental consent was also obtained.

\section{Competing interests}

The authors declare that they have no competing interests.

\section{Authors' contributions}

TS: Prepared manuscript, initial primary gastroenterologist and endoscopist. VN: Pathologist implicated in reviewing the histology. FC: Reviewed video capsule endoscopy literature. AS: Read the follow up capsule studies. ES: Helped in preparation of manuscript, endoscopist, read the video capsule studies and current primary gastroenterologist. All authors read and approved the final manuscript.

\section{Author details}

${ }^{1}$ Division of Pediatric Gastroenterology, Montreal Children's Hospital, Faculty of Medicine, McGill University, 2300 Tupper St, Montreal, Quebec H3H 1P3, Canada. ${ }^{2}$ Department of Pathology, Montreal Children's Hospital, Faculty of Medicine, McGill University, 2300 Tupper St, Montreal, Quebec H3H 1P3, Canada.

Received: 21 April 2013 Accepted: 30 December 2013 Published: 13 February 2014

\section{References}

1. Hill ID, Dirks MH, Liptak GS, Colletti RB, Fasano A, Guandalini S, Hoffenberg EJ, Horvath K, Murray JA, Pivor M, Seidman EG, North American Society for Pediatric Gastroenterology, Hepatology and Nutrition: Guideline for the diagnosis and treatment of celiac disease in children: recommendations of the North American society for pediatric gastroenterology, hepatology and nutrition. J Pediatr Gastroenterol Nutr 2005, 40(1):1-19.

2. Elsing C, Placke J, Gross-Weege W: Ulcerative jejunoileitis and enteropathy-associated T-cell lymphoma. Eur J Gastroenterol Hepatol 2005, 17(12):1401-1405.

3. Biagi F, Lorenzini P, Corazza CR: Literature review on the clinical relationship between ulcerative jejunoileitis, celiac disease, and enteropahty-associated T-cell lymphoma. Scand J Gastroenterol 2000, 35(8):785-790.

4. Dickson BC, Streutker CJ, Chetty R: Coeliac disease: an update for the pathologist. J Clin Pathol 2006, 59:1008-1016.
5. Green PH, Cellier C: Celiac disease. New Engl J Med 2007, 357:1731-1743.

6. Cellier C, Green PHR, Collin P, Murray J: ICCE concensus for celiac disease. Endocsopy 2005, 37(10):1055-1059.

7. Catassi C, Fabiani E, Rätsch IM, Coppa GV, Giorgi PL, Pierdomenico R, Alessandrini S, Iwanejko G, Domenici R, Mei E, Miano A, Marani M, Bottaro G, Spina M, Dotti M, Montanelli A, Barbato M, Viola F, Lazzari R, Vallini M, Guariso G, Plebani M, Cataldo F, Traverso G, Ventura A, et al: The coeliac iceberg in Italy. A multicentre antigliadin antibodies screening for coeliac disease in school-age subjects. Acta Paediatr Suppl 1996, 412:29-35.

8. Mulder CJJ, Cellier C: Coeliac disease: changing views. Best Pract Res Clin Gastroenterol 2005, 19(3):313-321.

9. Ho-Yen C, Chang F, van der Walt J, Mitchell T, Ciclitira P: Recent advances in refractory coeliac disease: a review. Histopathology 2009, 54(7):783-795.

10. Barret M, Malamut G, Rahmi G, Samaha E, Edery J, Verkarre V, Macintyre E, Lenain E, Chatellier G, Cerf-Bensussan N, Cellier C: Diagnostic yield of capsule endoscopy in refractory celiac disease. Am J Gastroenterol 2012, 107(10):1546-1553.

11. Proctor $D$, Panzini $L$ : Isolated and diffuse ulcers of the small intestine. In Sleisenger \& Fordtran's Gastrointestinal and Liver Disease. Volume 2. 7th edition. Edited by Feldman M, Friedman L, Sleisenger M. Philadelphia: Saunders; 2002:2081-2082.

12. Fritscher-Ravens A, Scherbakov P, Bufler P, Torroni F, Ruuska T, Nuutinen $H_{\text {, }}$ Thomson M, Tabbers M, Milla P: The feasibility of wireless capsule endoscopy in detecting small intestinal pathology in children under the age of 8 years: a multicenter European study. Gut 2009, 58:1467-1472.

13. O'Mahony S, Howdle PD, Losowsky MS: Review article: management of patients with non-responsive coeliac disease. Aliment Pharmacol Ther 1996, 10(5):671-680.

14. Daum S, Cellier C, Mulder C: Refractory coeliac disease. Best Pract Res Clin Gastroenterol 2005, 19(3):413-424.

15. Tennyson CA, Green PH: The role of capsule endoscopy in patients with nonresponsive celiac disease. Gastrointest Endosc 2011, 74(6):1323-1324.

16. Wahab PJ, Meijer JW, Mulder CJ: Histological follow-up of people with celiac disease on a gluten-free diet. Am J Clin Pathol 2002, 118(3):459-463.

17. Cellier C, Delabesse E, Helmer C, Patey N, Matuchansky C, Jabri B, Macintyre E, Cerf-Bensussan N, Brousse N: Refractory sprue, coeliac disease, and enteropathy-associated T-cell lymphoma. French Coeliac Disease Study Group. Lancet 2000, 356(9225):203-208.

18. Cellier C, Patey N, Mauvieux L, Jabri B, Delabesse E, Cervoni JP, Burtin ML, Guy-Grand D, Bouhnik Y, Modigliani R, Barbier JP, Macintyre E, Brousse N, Cerf-Bensussan N: Abnormal intestinal intraepithelial lymphocytes in refractory sprue. Gastroenterology 1998, 114(3):471-481.

19. Bagdi E, Diss TC, Munson P, Isaacson PG: Mucosal intra-epithelial lymphocytes in enteropathy-associated T-cell lymphoma, ulcerative jejunitis, and refractory celiac disease constitute a neoplastic population. Blood 1999, 94(1):260-264.

20. Daum S, Weiss D, Hummel M, Ullrich R, Heise W, Stein H, Riecken EO, Foss HD, Intestinal Lymphoma Study Group: Frequency of clonal intraepithelial T lymphocyte proliferations in enteropathy-type intestinal T cell lymphoma, coeliac disease, and refractory sprue. Gut 2001, 49(6):804-812.

21. Mubarak A, Oudshoorn JH, Kneepkens CM, Butler JC, Schreurs MW, Mulder CJ, Houwen RH: A child with refractory coeliac disease. J Pediatr Gastroenterol Nutr 2011, 53(2):216-218.

22. Goerres MS, Meijer JW, Wahab PJ, Kerckhaert JA, Groenen PJ, Van Krieken $\mathrm{JH}$, Mulder $\mathrm{CJ}$ : Azathioprine and prednisone combination therapy in refractory coeliac disease. Aliment Pharmacol Ther 2003, 18(5):487-494.

23. Tack GJ, van Asseldonk DP, van Wanrooij RLJ, van Bodegraven AA, Mulder $\mathrm{CJ}$ : Tioguanine in the treatment of refractory coeliac disease - a single centre experience. Aliment Pharmacol Ther 2012, 36:274-281.

24. Al-Toma A, Verbeek WH, Mulder CJ: The management of complicated celiac disease. Dig Dis 2007, 25(3):230-236.

25. Jantunen E, Boumendil A, Finel H, Luan J-J, Rambaldi A, et al: Autologous stem cell transplantation for enteropathy-associated T-cell lymphoma: a retrospective study by the EBMT. Blood 2013, 121(13):2529-2532.

26. Verbeek WH, Goerres MS, von Blomberg BM, Oudejans JJ, Scholten PE, Hadithi M, Al-Toma A, Schreurs MW, Mulder CJ: Flow cytometric determination of aberrant intra-epithelial lymphocytes predicts T-cell lymphoma development more accurately than T-cell clonality analysis in refractory celiac disease. Clin Immunol 2008, 126(1):48-56.

doi:10.1186/1471-230X-14-29

Cite this article as: Sigman et al:: Ulcerative jejunitis in a child with celiac disease. BMC Gastroenterology 2014 14:29. 